ORIGINAL ARTICLE

\title{
Eicosapentaenoic acid ameliorates hyperglycemia in high-fat diet-sensitive diabetes mice in conjunction with restoration of hypoadiponectinemia
}

\author{
M Morimoto ${ }^{1,2,5}$, E-Y Lee ${ }^{1,5}$, X Zhang ${ }^{1}$, Y Inaba ${ }^{3}$, H Inoue $^{3}$, M Ogawa $^{2}$, T Shirasawa ${ }^{4}$, O Yokosuka ${ }^{2}$ and T Miki $^{1}$
}

BACKGROUND/OBJECTIVE: Eicosapentaenoic acid (EPA) exerts pleiotropic effects on metabolic disorders such as atherosclerosis and dyslipidemia, but its effectiveness in the treatment of type 2 diabetes mellitus remains controversial. METHODS: We examined the antidiabetic effect of EPA in insulin receptor mutant $\left(I n s r^{P 1195 L /+}\right)$ mice that exhibit high-fat diet (HFD)-dependent hyperglycemia.

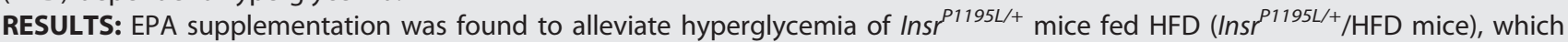
was accompanied by amelioration of increased gluconeogenesis and impaired insulin signaling, as assessed by glucose-6phosphatase (G6pc) expression on refeeding and insulin-induced phosphorylation of Akt in the liver, respectively. We found that serum levels of adiponectin, the antidiabetic adipokine, were decreased by HFD along with the body weight gain in Insr ${ }^{P 1195 L /+}$ mice but not in wild-type mice, suggesting that $I n s r^{P 1195 L /+}$ mice are prone to hypoadiponectinemia in response to obesity. Interestingly, the blood glucose levels of $I n s r^{P 1195 / /+}$ mice were in reverse proportion to their serum adiponectin levels and EPA supplementation ameliorated their hyperglycemia in conjunction with the restoration of hypoadiponectinemia.

CONCLUSIONS: EPA exerts an antidiabetic effect in Insr ${ }^{P 1195 L /+} / \mathrm{HFD}$ mice, an HFD-sensitive, insulin-resistant animal model, possibly through its action against hypoadiponectinemia.

Nutrition \& Diabetes (2016) 6, e213; doi:10.1038/nutd.2016.21; published online 27 June 2016

\section{INTRODUCTION}

Type 2 diabetes mellitus (T2DM) is a metabolic disorder characterized by chronic hyperglycemia, and the number of patients with T2DM has been increasing worldwide. Many environmental factors such as overnutrition and sedentary lifestyle are considered to be responsible for this increase. ${ }^{1}$ Especially, excessive intake of fat rich in saturated fatty acid is considered to be associated with the development of insulin resistance and consequential T2DM. ${ }^{2}$ Recently, we found that high-fat diet (HFD) feeding of a mouse harboring a loss-of-function mutation in the insulin receptor $\left(\right.$ Insr $^{P 1195 L /+}$ ) developed overt hyperglycemia. ${ }^{3}$ This mutation is a single amino-acid substitution from proline to leucine at 1195 amino-acid residue (P1195L), which acts as a dominant-negative mutant in the heterozygote. In Insf ${ }^{P 195 L /+}$ mice fed HFD (Insr ${ }^{P 1195 L /+} /$ HFD mice), lipolysis in white adipose tissue (WAT) and gluconeogenesis in the liver were both increased, and these are considered to be responsible for hyperglycemia. Lipolysis of triglyceride (TG) generates fatty acids and glycerol. Fatty acids are classified into three groups: saturated, monounsaturated, and polyunsaturated (PUFA) fatty acids. PUFA is further subclassified into $n-6$ PUFA and $n-3$ PUFA. The latter has a double bond at the third carbon from the methyl end of the carbon chain. The HFD used in our study contains a high percentage of saturated fatty acid, which is known to induce chronic inflammation in WAT, worsening insulin resistance. ${ }^{4}$ By contrast, $n-3$ PUFAs such as eicosapentaenoic acid (EPA) and docosahexaenoic acid have been reported to suppress chronic inflammation through various pathways involving Toll-like receptor 4, resolvin E1 and GRP120. ${ }^{5-7}$

In the present study, we hypothesized that chronic inflammation in WAT might contribute to the development of hypergly-

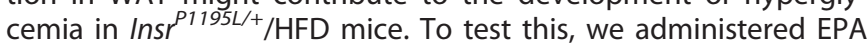
to Insr ${ }^{P 1195 L /+} /$ HFD mice and evaluated chronic inflammation and glucose metabolism in these mice. Although chronic inflammation was not increased in $/ n s r^{P 1195 L /+} /$ HFD mice compared with that in wild-type (WT)/HFD mice, EPA supplementation significantly ameliorated hyperglycemia of $I n s r^{P 1195 L /+} / \mathrm{HFD}$ mice. In the present study, we examined the underlying mechanism for improved hyperglycemia in Insr $^{\text {P1195L/+ } / H F D ~ m i c e ~ b y ~ E P A . ~}$

\section{MATERIALS AND METHODS}

\section{Animals}

Mice were allowed free access to water and laboratory chow. All mouse experiments except for Figures $1 \mathrm{a}$ and $\mathrm{b}$ were performed in male mice aged 16-18 weeks. They were kept under 12-h light/12-h dark cycle. For normal diet (ND) feeding and for feeding before the initiation of HFD, mice were maintained on standard chow (CE-2) $(12.1 \%$ kcal from fat; Clea Japan Inc., Tokyo, Japan). InsP ${ }^{P 195 L /+}$ /HFD and WT/HFD mice with C57BL/6 background were raised as described previously. ${ }^{3}$ Briefly, mice were maintained on a HFD (D12492) $(60.0 \%$ kcal from fat) (Research Diets Inc., New Brunswick, NJ, USA) starting at 8 weeks of age unless stated otherwise. For EPA supplementation,

\footnotetext{
'Department of Medical Physiology, Chiba University, Graduate School of Medicine, Chiba, Japan; ${ }^{2}$ Department of Gastroenterology and Nephrology, Chiba University, Graduate School of Medicine, Chiba, Japan; ${ }^{3}$ Metabolism and Nutrition Research Unit, Innovative Integrated Bio-Research Core, Institute for Frontier Science Initiative, Kanazawa University, Kanazawa, Japan and ${ }^{4}$ Department of Neurology, University of Michigan, Ann Arbor, MI, USA. Correspondence: Professor T Miki, Department of Medical Physiology, Chiba University, Graduate School of Medicine, 1-8-1 Inohana, Chuo-ku, Chiba 260-8670, Japan.

E-mail: tmiki@faculty.chiba-u.jp

${ }^{5}$ These authors contributed equally to this work.

Received 11 December 2015; revised 11 April 2016; accepted 24 May 2016
} 
a

O WT/HFD (13)
- WT/HFD+EPA (14)

$\triangle I n s r^{P 1195 L+/ H F D ~(19)}$

$\Delta$ Insr $^{P 1195 L+/ H F D+E P A ~(20) ~}$

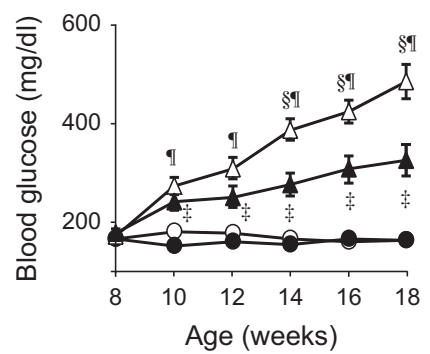

d $O$ WT/HFD (8)

- WT/HFD+EPA (9)

$\triangle$ Insr $P^{P 195 L+} / \mathrm{HFD}(7)$

$\triangle I n s r^{P 1195 L+/ H F D+E P A ~(8) ~}$

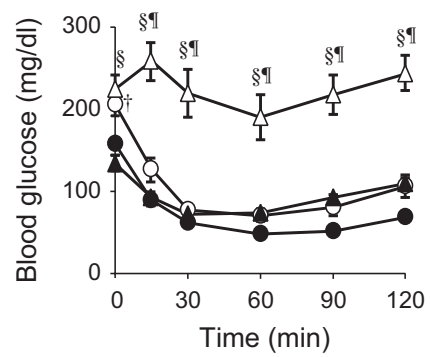

g $\bigcirc$ WT/HFD (10)

- WT/HFD+EPA (7)

$\triangle I n s r^{P 195 L+/ H F D ~(8)}$

$\triangle I n s r^{P 1195 L+/ H F D+E P A ~(11) ~}$

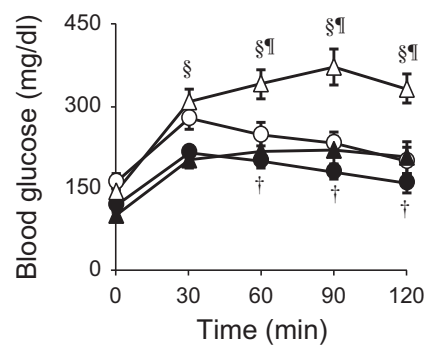

b

O WT/HFD (13)

- WT/HFD+EPA (14)

$\triangle I n s r^{P 1195 L+/ H F D ~(19) ~}$

$\Delta$ Insr $^{P 1195 L+/ H F D+E P A ~(20) ~}$

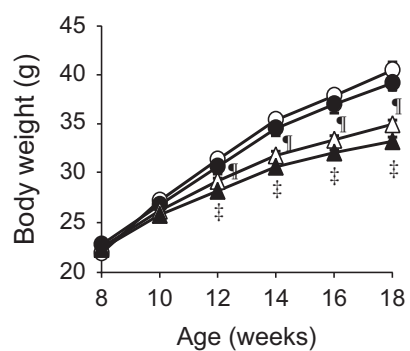

e

$$
\square \text { Fasted }
$$

- Refed

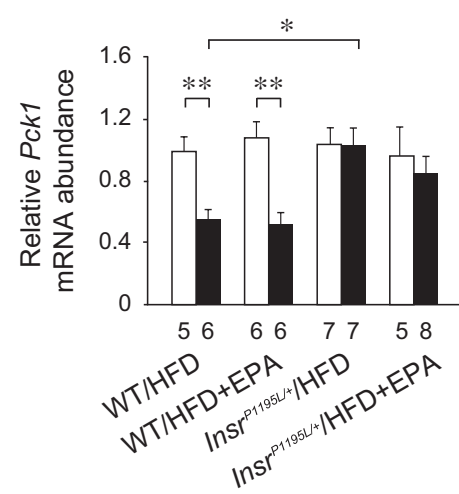

C

O WT/HFD (11)

- WT/HFD+EPA (7)

$\triangle$ Insr $r^{P 1195 L+} / \mathrm{HFD}(8)$

$\Delta I n s r^{P 1195 L+} / \mathrm{HFD}+\mathrm{EPA}(12)$

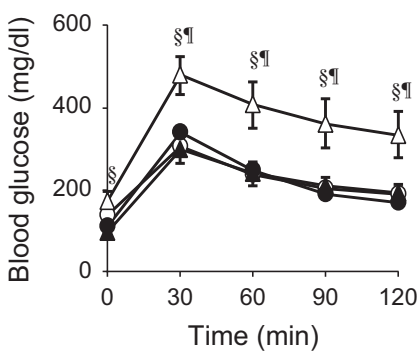

f

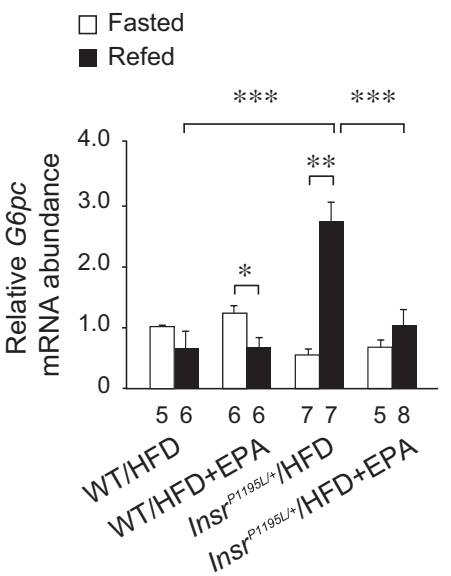

Figure 1. Amelioration of glucose metabolism by EPA supplementation. (a) Changes in blood glucose levels fed ad libitum. (b) Changes in body weight. (c) Blood glucose levels after oral glucose loading $\left(1 \mathrm{~g} \mathrm{~kg}^{-1}\right)$. (d) Blood glucose levels after intraperitoneal insulin administration $\left(0.75 \mathrm{U} \mathrm{kg}^{-1}\right)$. (e, f) mRNA expression levels of $P c k 1(\mathbf{e})$ and G6pc (f) in the liver at fasted (after $16 \mathrm{~h}$ fasting) and refed (after $3 \mathrm{~h}$ refeeding) state. ${ }^{*} P<0.05$, ${ }^{* *} P<0.01,{ }^{* * *} P<0.001$. (g) Changes in blood glucose levels after intraperitoneal glycerol administration $\left(0.5 \mathrm{~g} \mathrm{~kg}{ }^{-1}\right)$. Data are mean \pm s.e.

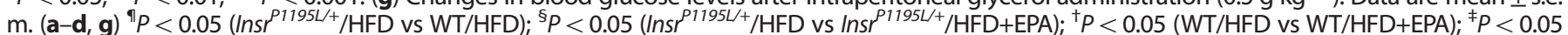
$\left(\right.$ Insi ${ }^{P 1195 L /} /$ HFD+EPA vs WT/HFD+EPA). The number in the parenthesis $(\mathbf{a}-\mathbf{d}, \mathbf{g})$ and in the bottom of the columns (e, $\left.\mathbf{f}\right)$ denotes sample size.

EPA (kindly provided by Mochida Pharmaceutical Co. Ltd., Tokyo, Japan) was added to the HFD ( $5 \% w t / w t)$ and was given to the mice starting at 8 weeks of age. Each type of chow was assigned randomly. Animal care and experiments were performed in accordance with the guidelines of Chiba University, Japan. No sample size estimation and blinding was carried out in the experiments.

Metabolic analysis and blood biochemistry

Glucose tolerance test (oral glucose tolerance test), insulin tolerance test and glycerol tolerance test were performed as previously described. ${ }^{8}$ Briefly, for oral glucose tolerance test, the mice were fasted for $16 \mathrm{~h}$ and glucose $\left(1 \mathrm{~g} \mathrm{~kg}^{-1}\right)$ was administered orally. For insulin tolerance test and glycerol tolerance test, 16 - $\mathrm{h}$-fasted mice were administered intraperitoneally with $0.75 \mathrm{IU} \mathrm{kg}{ }^{-1}$ insulin and $0.5 \mathrm{~g} \mathrm{~kg}^{-1}$ glycerol, respectively. Blood glucose was measured as previously described. ${ }^{8}$ Serum concentrations of total adiponectin (Otsuka pharmaceuticals, Tokyo, Japan) and free fatty acid (FFA) (BioVision, Inc., San Francisco, CA, USA) were measured using the commercially available kits. TG content in the liver was measured using the kit from BioVision, Inc.

Primers and quantitative reverse transcriptase-PCR

Total RNA was isolated from the WAT (epididymal fat pad) and liver and subjected to quantitative reverse transcriptase-PCR analyses using SYBR Green (Thermo Fisher Scientific, Waltham, MA, USA), according to the 
manufacturer's protocol. The primers used were $5^{\prime}$ CACCATCACCTCCTGGAAGA-3' and 5'-GGGTGCAGAATCTCGAGTTG-3' for Pck1, 5'-GTGGCAGTGGTCGGAGACT-3' and 5'-ACGGGCGTTGTCCAAAC-3' for G6pc, 5'-CGGAGTCCGGGCGGT-3' and 5'-GCTGGGTAGAGAATGGATGAA CA-3' for Tnfa, 5'-CTTTGGCTATGGGCTTCCAGTC-3' and 5'-GCAAGGAGG ACAGAGTTTATCGTG-3' for Emr1， 5'-AGAGATGGCACTCCTGGAGAGAA-3' and 5'- CAACATCTCCTGTCTCACCCTTA-3' for Acrp30, 5'-GTTITATGCT GTTATGGGTG-3' and 5'-GTAATTTCTTGTGAAGTGCTCATAG-3' for Pparg2 5'-TACAGAGTGCTGGCCAAGAG-3' and 5'-GCGTCGTGATTAGCGATGA-3' and 5'-ATGGCCTCCCATCTCCTT-3' for Hprt.

\section{Western blotting}

Akt phosphorylation in the liver was examined in the four mouse groups $\left(\right.$ Insr ${ }^{P 1195 L /+}$ and WT mice under either HFD or HFD+EPA) at 18 weeks of age (after 10 weeks of HFD feeding). The cervical vein was exposed and $0.1 \mathrm{IU} \mathrm{kg}^{-1}$ insulin was injected via the vein. Five minutes later, the liver was removed. Tissues were lysed in sonication buffer ( $20 \mathrm{~mm}$ HEPES pH7.5; $150 \mathrm{~mm} \mathrm{NaCl} ; 25 \mathrm{~mm}$ EDTA; 1\% NP-40; 10\% glycerol, $1 \mathrm{~mm}$ sodium vanadate, $1 \mathrm{~mm}$ phenylmethylsulfonyl fluoride and protease and phosphatase inhibitors). Fifty micrograms of protein was subjected to sodium dodecyl sulfate-polyacrylamide gel electrophoresis. Antibodies against the following proteins were used (all from Cell Signaling Technology, Danvers, MA, USA): phospho-Akt (p-S473) (1:1000, no. 9271), Akt (1:1000, no. 9272), and $\beta$-actin $(1: 1000$, no. 4967). Band intensities were quantified by the Image J Software (National Institutes of Health, Bethesda, MD, USA).

\section{Primary hepatocyte experiments}

Primary mouse hepatocytes were isolated as previously described. ${ }^{9}$ Briefly, the livers of 18-week-old male mice were perfused through the portal vein $\left(5 \mathrm{ml} \mathrm{min}{ }^{-1}\right.$ for $17 \mathrm{~min}$ at $37^{\circ} \mathrm{C}$ ) with $1 \times$ HBSS (WAKO, Osaka, Japan) supplemented with $10 \mathrm{~mm}$ HEPES (GIBCO, Grand Island, NY, USA), $0.4 \mathrm{mg} \mathrm{ml}^{-1}$ type 1 collagenase (CLS-1, Worthington, Lakewood, NJ, USA) and protease inhibitor cocktail (cOmplete, EDTA-free, Roche, Basel, Switzerland). After filtration and washing, hepatocytes were isolated using a $45 \%(v / v)$ Percoll (GE Healthcare, Buckinghamshire, UK) solution. The hepatocytes were resuspended in William's E medium (Sigma-Aldrich Co., St Louis, MO, USA) containing $10 \%$ fetal bovine serum, $100 \mathrm{~mm}$ dexamethazone, $10 \mathrm{~nm}$ insulin and $1 \times$ Penicillin-Streptomycin-L-Glutamine Solution (WAKO), plated to six-well plate $\left(5.0 \times 10^{5}\right.$ hepatocytes per well) and cultured for $10 \mathrm{~h}$. The medium was then changed to William's E medium containing $1 \%$ bovine serum albumin with or without $100 \mathrm{nM}$ EPA sodium salt (Sigma-Aldrich) and/or $5 \mu \mathrm{M}$ LY249002 (Sigma-Aldrich), and the cells were cultured for $24 \mathrm{~h}$. Hepatocytes were stimulated with or without $10 \mathrm{~nm}$ insulin for $5 \mathrm{~min}$, harvested and sonicated. Thirty micrograms of protein was subjected to western blotting.

\section{Histological examination}

Histological analyses were performed as previously described..$^{10}$ WAT and liver were dissected and fixed in $10 \%$ buffered formalin overnight and embedded in paraffin. Sections $(4 \mu \mathrm{m})$ were stained with hematoxylin and eosin (HE) for histological analysis. For immunofluorescence staining of caveolin, sections were incubated in $1.5 \%$ goat serum dissolved in PBS-T (phosphate-buffered saline containing $0.3 \%$ Tween-20) for $1 \mathrm{~h}$ at room temperature. Sections were stained with rabbit anti-caveolin antibody $(1: 500$, no. $610060, \mathrm{BD}$ Biosciences, Franklin Lakes, NJ, USA) overnight at $4{ }^{\circ} \mathrm{C}$. Subsequently, incubation with secondary antibody (donkey anti-rabbit lgG antibody conjugated with Alexa-Fluor 555, 1:1000, Thermo Fisher Scientific) was performed for $90 \mathrm{~min}$ at room temperature. For imaging, slides were examined under a Biozero fluorescence microscope (BZ-8100, Kyence, Osaka, Japan).

\section{Statistical analyses}

Results are expressed as means \pm s.e.m. Data were analyzed using the statistical software package, IBM SPSS Version 19 (IBM Corporation, Armonk, NY, USA). After confirming all variables to be normally distributed, differences between two groups were assessed using the unpaired two-tailed Student's $t$-test. Data sets involving more than three groups were assessed by one-way analysis of variance with Bonferroni post-hoc test. $P<0.05$ was considered to be statistically significant.

\section{RESULTS}

EPA ameliorated hyperglycemia in $/ n s r^{P 1195 L /+}$ mice fed HFD Insr $^{P 1195 L /+}$ mice under HFD (Insr ${ }^{P 1195 L /+} /$ HFD mice) developed hyperglycemia as previously reported. ${ }^{3}$ However, EPA supplementation significantly improved hyperglycemia of Insr $^{P 195 L /+} / \mathrm{HFD}$ mice from 14 to 18 weeks of age (Figure 1a). In addition, EPAsupplemented-Insr ${ }^{P 1195 L /+} /$ HFD mice $\left(I n s r^{P 1195 L /+} /\right.$ HFD+EPA mice) exhibited modestly reduced body weight gain compared with Insr $^{P 1195 L /+} /$ HFD mice (Figure 1b). By contrast, EPA supplementation failed to evoke significant decrease in either body weight gain or blood glucose levels in WT mice. Glucose tolerance in Insr $P^{\text {1195L/+ } / H F D ~}$ mice was markedly impaired (Figure 1c). However, EPA supplementation significantly attenuated the blood glucose rise after oral glucose loading. In addition, EPA supplementation improved insulin sensitivity in Insr $^{P 1195 L /+} / \mathrm{HFD}$ mice, as assessed by insulin tolerance test (Figure 1d). The reduction in blood glucose levels of Insr $^{P 1195 L /+} /$ HFD mice by EPA supplementation became statistically insignificant at or later than 24 weeks of age (data not shown). Therefore, all the experiments were performed in the mice aged 16-18 weeks, unless otherwise specified.

We then examined whether EPA suppresses gluconeogenesis by quantifying the expression of gluconeogenesis-related genes, such as phosphoenolpyruvate carboxykinase 1 (Pck1) and glucose-6phosphatase (G6pc). EPA supplementation did not affect Pck1 expression in both WT/HFD and Insr $P^{P 1195 L /+} /$ HFD mice (Figure 1e). G6pc expression in Ins $r^{P 1195 L /+} /$ HFD mice was markedly increased after $3 \mathrm{~h}$ refeeding (Figure $1 \mathrm{f}$ ), as previously reported, ${ }^{3}$ while EPA supplementation significantly suppressed G6pc expression in Insr $^{P 1195 L /+} /$ HFD mice. G6pc is a key enzyme involved in gluconeogenesis from glycerol as well as from amino acids and pyruvate. We therefore examined gluconeogenesis by measuring the blood glucose rise after intraperitoneal glycerol administration (Figure 1g). Although gluconeogenesis from glycerol was increased in Insr $^{P 1195 L /+} /$ HFD mice, as previously reported, ${ }^{3}$ the blood glucose rise after glycerol administration was significantly attenuated in $\mathrm{Insr}^{\text {P1195L/+}} / \mathrm{HFD+EPA}$ mice, suggesting that EPA supplementation may ameliorate hyperglycemia through suppression of G6pc expression.

\section{Akt phosphorylation by insulin was improved by EPA} supplementation in $\operatorname{Insr}^{P 1195 L /+} /$ HFD mice

Insulin is known to inhibit gluconeogenesis in hepatocytes. As gluconeogenesis has been shown to be increased in Insr P1195L/+ /HFD mice, we first evaluated insulin signaling in the liver in vivo by examining Akt phosphorylation after insulin administration $\left(0.1 \mathrm{U} \mathrm{kg}^{-1}\right)$ to jugular vein (Figures $2 \mathrm{a}$ and b). Quantification of phosphorylated Akt (p-Akt) revealed that insulin-induced Akt phosphorylation was significantly impaired in $/ \mathrm{ns}^{\mathrm{P} 1195 \mathrm{~L} /+} / \mathrm{HFD}$ mice compared with that in WT/HFD, as previously described. ${ }^{3}$ In WT/HFD mice, EPA supplementation did not affect insulin-induced Akt phosphorylation. Although insulin-induced Akt phosphorylation was blunted in $\operatorname{Ins}^{P 1195 L /+} / \mathrm{HFD}$ mice, EPA supplementation suppressed the basal p-Akt levels and was considered to restore the responsiveness of Akt phosphorylation to insulin in these mice.

We next examined whether EPA directly activates the insulin signaling cascade in primary hepatocytes. For this purpose, primary hepatocytes were isolated from $/ n r^{P 1195 L /+} / H F D$ and WT/HFD mice, precultured in the presence or absence of EPA and subjected to stimulation with insulin (Figures $2 c$ and d). In the absence of EPA, insulin significantly stimulated Akt phosphorylation in WT/HFD hepatocytes but not in Insr ${ }^{P 1195 L /+} / \mathrm{HFD}$ hepatocytes. EPA treatment partially ameliorated the insulin responsiveness in Insr $P^{1195 L /+} / \mathrm{HFD}$ hepatocytes, which resembles the trend of $p$-Akt by insulin administration in vivo (Figures $2 \mathrm{a}$ and $\mathrm{b}$ ).

In search for the mechanism of EPA action on Akt phosphorylation, the primary hepatocytes of WT mice under ND (WT/ND mice) were pretreated with or without a phosphoinositide 3-kinase 
a p-Akt (S473)

Akt

ß-actin
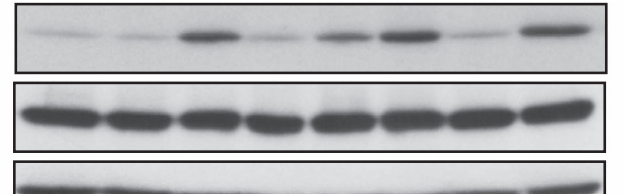

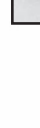

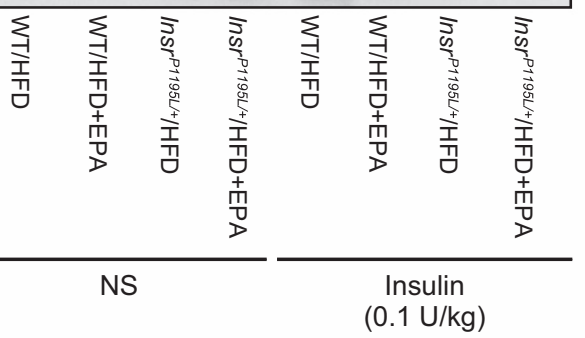

C

p-Akt (S473)

Akt

ß-actin

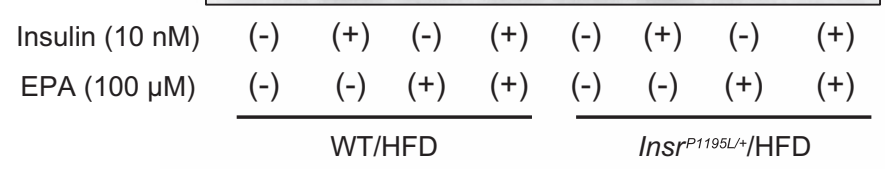

e p-Akt (S473)

Akt

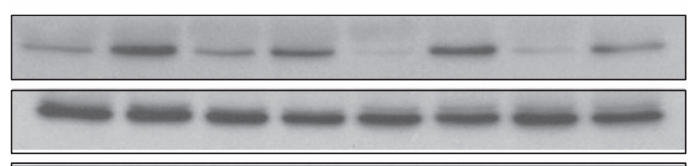

ß-actin

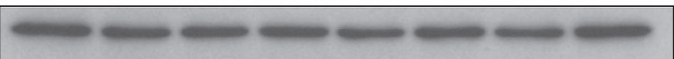

\section{Insulin (10 nM)}

$(-) \quad(+)$

$(-) \quad(+)$

$(-)$

(+) (-)

(+)

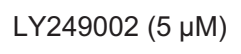

$(-)$

(-)

(+) (+)

$(-)$

$(-) \quad(+)$

(+)

EPA $(100 \mu \mathrm{M})$

$(-)$

(-)

$(-) \quad(+)$

(+) (+)

b

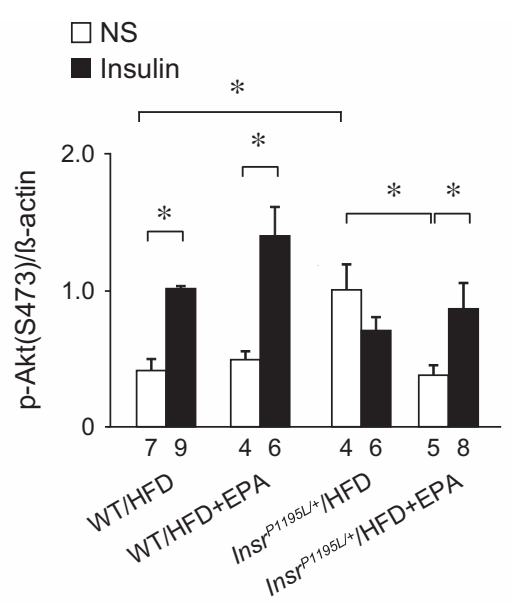

d

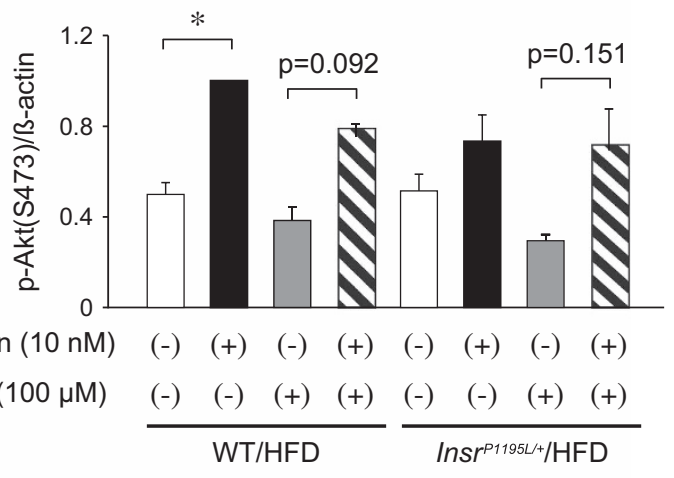

f

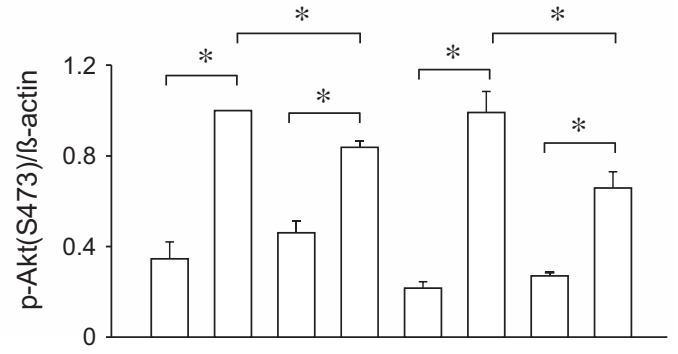
Insulin (10 nM)
$(-) \quad(+)(-)$
$(+) \quad(-)$
$(+) \quad(-) \quad(+)$
LY249002 (5 $\mu \mathrm{M})$
$(-) \quad(-)$
(+) $(+$
$(-)$
$(-) \quad(+) \quad(+)$
EPA $(100 \mu \mathrm{M})$
$(-) \quad(-)$
(-) (-)
$(+) \quad(+)$
) $(+)(+)$

Figure 2. Phosphorylation of Akt in the liver and primary hepatocytes. (a, b) Akt phosphorylation after insulin administration $\left(0.1 \mathrm{U} \mathrm{kg}^{-1}\right.$ intravenous) in vivo. (c-f) Akt phosphorylation in primary hepatocytes. (a, c, e) A representative result of p-Akt. (b) Quantified result of p-Akt levels. The number in the bottom of the columns denotes sample size. (d, f) Quantified result of p-Akt levels ( $n=3$ per each group). Data are mean \pm s.e.m. ${ }^{*} P<0.05$.

(PI3K) inhibitor LY294002 and insulin-induced Akt phosphorylation was examined (Figures 2e and f). EPA treatment did not affect insulin-induced Akt phosphorylation but rather tended to decrease its basal level. On the contrary, LY294002 suppressed the insulininduced Akt phosphorylation similarly in the EPA-treated and EPA-untreated hepatocytes. Thus EPA supplementation in vivo may recapitulate the defective insulin-induced Akt phosphorylation in the Insr ${ }^{P 1195 L /+} / \mathrm{HFD}$ liver (Figure 2b) by lowering the basal p-Akt level through a PI3K-independent pathway.

EPA modestly suppressed WAT inflammation of $/ n s r^{P 1195 L /+} / \mathrm{HFD}$ mice

As EPA is well known to improve insulin sensitivity via its antiinflammatory action in WAT, $^{11}$ we examined the inflammatory changes in WAT of Insr ${ }^{P 1195 L /+} /$ HFD+EPA mice. Chronic inflammation in WAT evokes morphological changes called crown-like structures (CLS), which are composed of macrophages surrounding dying or dead adipocytes. ${ }^{12}$ Histological examination by HE staining in WAT revealed that the number of CLS was significantly increased by HFD feeding (Figures $3 a$ and b). However, the frequency of CLS was not different between Insr $^{P 1195 L /+} / \mathrm{HFD}$ and WT/HFD mice and was not decreased by EPA supplementation.

We measured the serum FFA, a surrogate marker of lipolysis and inflammation ${ }^{4}$ and found that they were significantly increased by HFD (Figure 3c). There was no significant difference in FFA levels between Insr $^{P 1195 L /+} / \mathrm{HFD}$ and WT/HFD mice, and EPA supplementation significantly lowered FFA in Insr $^{P 1195 L /+} /$ HFD mice.

We also examined the change in the mRNA expression levels of two inflammatory-related genes; Tnfa and Emr1, which encode 
a

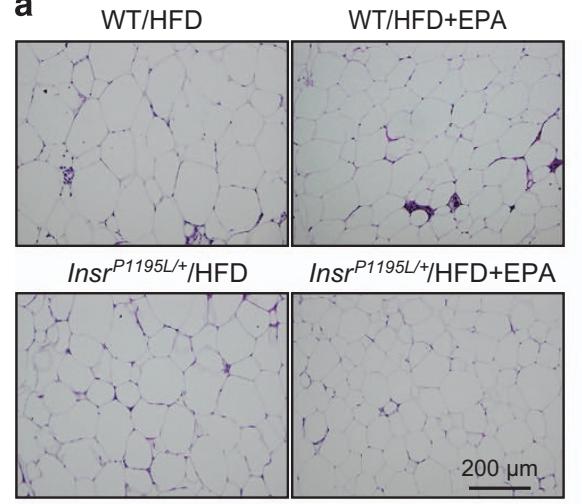

b

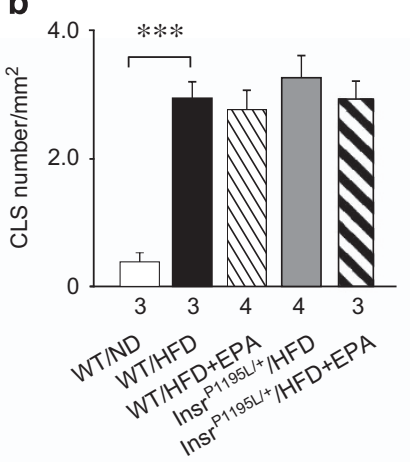

C
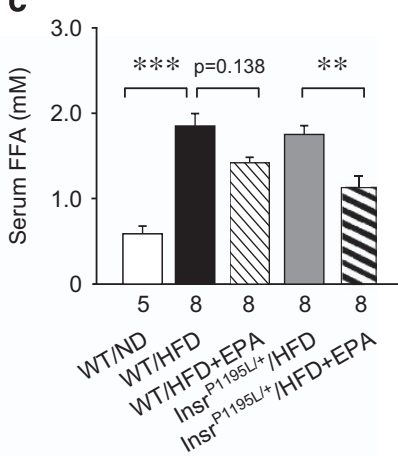

d

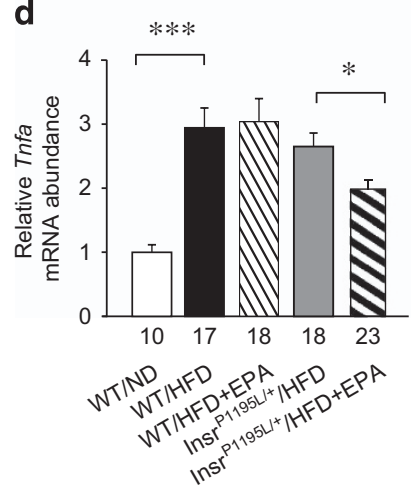

e

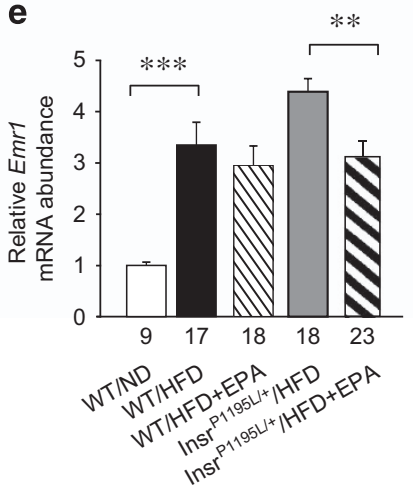

Figure 3. Chronic inflammation in WAT of $I n s r^{P 1195 L /+} / H F D$ mice. (a, b) HE staining of epididymal fat. (a) A representative result. Scale bar: $200 \mu \mathrm{m}$. (b) CLS number. (c) Serum FFA levels. (d, e) mRNA expression levels of Tnfa (d) and Emr1 (e). Data are mean \pm s.e.m. ${ }^{*} P<0.05$, ${ }^{* *} P<0.01,{ }^{* *} P<0.001$. (b-e) The number in the bottom of the columns denotes sample size.

tumor necrosis factor $a$ and EGF (epidermal growth factor)-like module-containing mucin-like hormone receptor-like 1 (also known as F4/80), respectively (Figures $3 \mathrm{~d}$ and e). HFD feeding of WT mice significantly increased the expression levels of these genes, but there was no significant difference between Inst $P^{9195 L /+} / \mathrm{HFD}$ and WT/HFD mice. By contrast, EPA supplementation significantly suppressed these expression levels in $\operatorname{Insr}^{P 1195 L /+} / \mathrm{HFD}$ mice. Accordingly, EPA supplementation may ameliorate the hyperglycemia of InsP ${ }^{1195 L /+} /$ HFD mice, at least in part, by attenuating chronic inflammation.

EPA supplementation reduced adipocyte size in WAT and adiposity in vivo

HE staining revealed that WAT in EPA-supplemented mice contained a number of small adipocytes in both WT/HFD and Insr $^{P 1195 L /+} /$ HFD mice. We therefore performed computed tomography scanning of the four mouse groups (Figure 4a). As previously reported, ${ }^{3}$ the visceral fat area in Insr ${ }^{P 195 L /+} /$ HFD mice was reduced when compared with that in WT/HFD mice. In addition, computed tomography scanning suggested that adiposity might be reduced mildly by EPA supplementation. We also measured epididymal fat weight and found that it was significantly decreased by EPA supplementation in Insr $^{P 1195 L /+} /$ HFD mice (Figure 4b).

We then quantified adipocyte size by immunostaining of caveolin in WAT (Figures 4C-e). The average adipocyte size in Insr ${ }^{P 195 L /+} / \mathrm{HFD}$ mice tended to be smaller than that in WT/HFD mice $(P=0.10$; Figure $4 d)$, and EPA supplementation significantly reduced the average adipocyte size in both WT/HFD and Insr ${ }^{P 195 L /+} /$ HFD mice. Notably, EPA supplementation decreased the number of large $\left(>10000 \mu \mathrm{m}^{2}\right)$ adipocytes in both WT and Insr ${ }^{P 1195 L /+}$ mice (Figure 4e). In addition to its effect on WAT, EPA supplementation ameliorated the steatosis in the liver of WT/HFD mice as assessed by HE staining (Figure $4 f$ ) and TG content in the liver (Figure 4g). In Insr ${ }^{P 195 L /+} /$ HFD mice, the TG content was much lower than that in WT/HFD mice, and there was no further decrease by EPA supplementation.

Insr ${ }^{P 1195 L /+}$ mice were susceptible to body weight-dependent reduction in serum adiponectin and EPA supplementation restored the reduction

As adiposity is known to be negatively correlated with serum adiponectin levels in humans and rodents, ${ }^{13}$ we examined serum adiponectin levels (Figure 5a). In this analysis, we also included the data of WT/ND and Insr $r^{P 1195 L /+} /$ ND mice. The serum adiponectin levels in WT/HFD mice were not different from those in WT/ND mice and EPA supplementation did not change them. By contrast, serum adiponectin levels in $\mathrm{Insr}^{P 1195 L /+} / \mathrm{HFD}$ mice were significantly lower than those in Insr ${ }^{P 1195 L /+} /$ ND mice. In addition, EPA supplementation significantly increased serum adiponectin levels in Insr $^{P 1195 L /+} /$ HFD mice. We then evaluated correlation between body weight and adiponectin levels (Figure 5b) and between blood glucose levels and adiponectin levels (Figure 5c). Interestingly, we found the negative correlation between body weight and adiponectin levels in InsP ${ }^{P 1195 L /+}$ mice $(r=-0.502, P<0.001)(n=76)$ but not in WT $(n=70)$ mice. EPA supplementation decreased body weight and increased adiponectin levels in Inst $P^{1195 L /+} / \mathrm{HFD}$ mice. These data suggest that $I n r^{P 1195 L /+}$ mice are susceptible to hypoadioponectinemia in response to obesity. Furthermore, there was a clear negative correlation between blood glucose levels and adiponectin levels in all the experimental groups, including WT and Insr ${ }^{P 1195 L+}$ mice $(r=-0.628, P<0.001)(n=146)$. EPA supplementation increased adiponectin levels proportionally to the decrease in blood glucose levels in InsP ${ }^{P 1195 L /+} / \mathrm{HFD}$ mice, suggesting that EPA supplementation improves hyperglycemia through the action of adiponectin. 
To investigate the mechanism of the increase of serum adiponectin levels by EPA, mRNA expression of adiponectin was quantified. The mRNA expression levels of adiponectin were significantly increased by EPA supplementation in Insr ${ }^{P 1195 L /+} / \mathrm{HFD}$ mice (Figure $5 \mathrm{~d}$ ). Interestingly, mRNA expression of Pparg2, which is known to be transactivated by insulin, ${ }^{14}$ also was changed by a

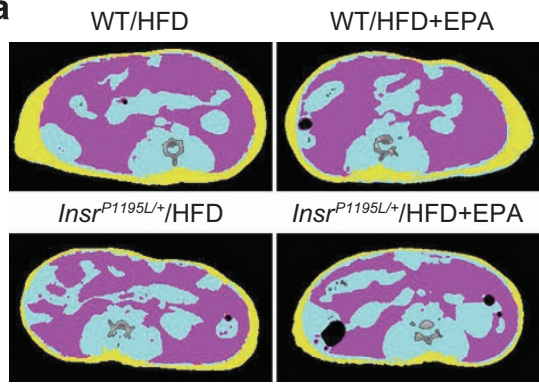

C

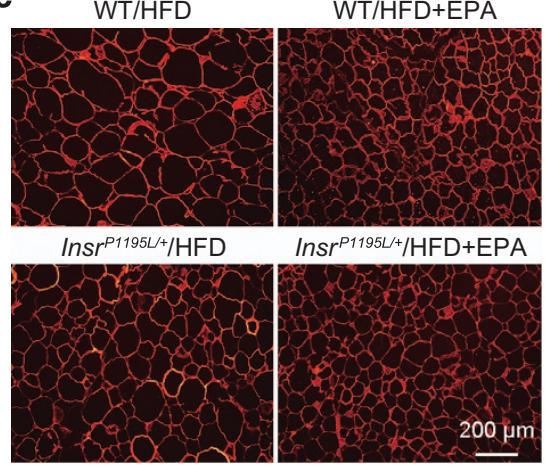

e

WT/HFD+EPA (5)

$\square \quad I n s r^{P 1195 L+/ H F D ~(5) ~}$

\Insr ${ }^{P 1195 L+} / \mathrm{HFD}+\mathrm{EPA}(7)$

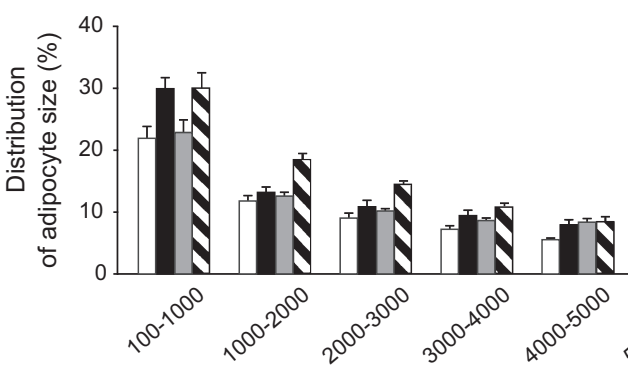

$\mathbf{f}$

WT/HFD

WT/HFD+EPA

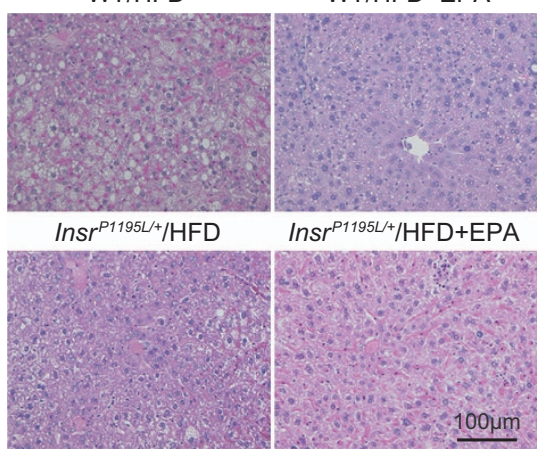

b
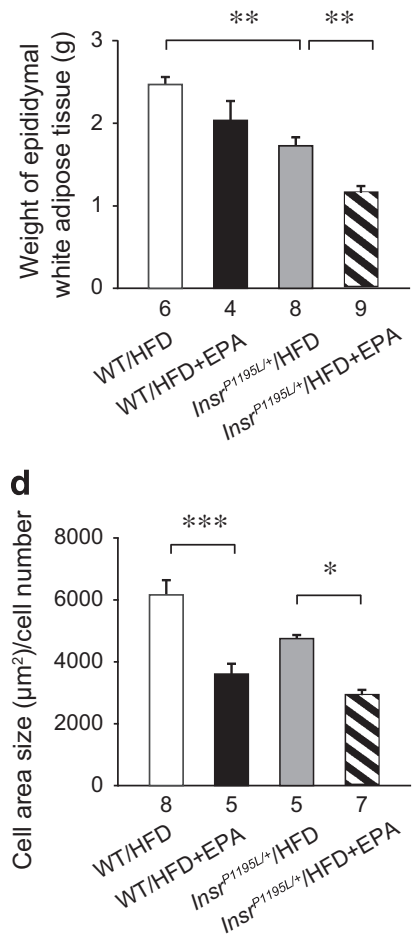

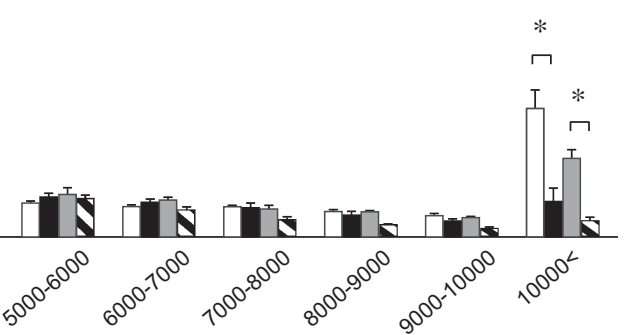

g

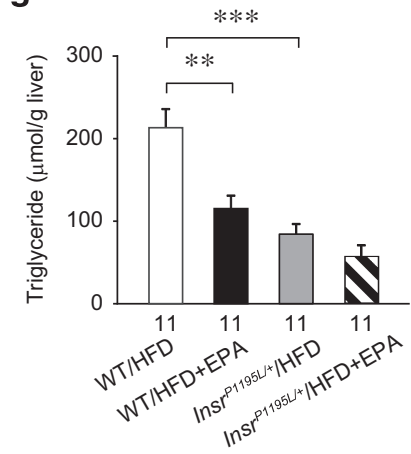

Figure 4. Effect of EPA supplementation on adiposity and steatosis in Insr ${ }^{P 1195 L /+} /$ HFD mice at $18-20$ weeks of age. (a) Computed tomographic images at the level of lower end of right kidney. Visceral and subcutaneous fat are indicated in pink and yellow, respectively. (b) Weight of epididymal WAT. (c) Immunostaining of caveolin in epididymal fat. (d) Mean adipocyte size. Scale bar: $200 \mu \mathrm{m}$. (e) Histogram of the cell area of four mouse groups. (f) HE staining of the liver. (g) TG content in the liver. (b, d, e, g) Data are mean \pm s.e.m. ${ }^{*} P<0.05,{ }^{* *} P<0.01,{ }^{* * *} P<0.001$. The number in the bottom of the columns $(\mathbf{b}, \mathbf{d}, \mathbf{g})$ and in the parenthesis $(\mathbf{e})$ denotes sample size. 
a
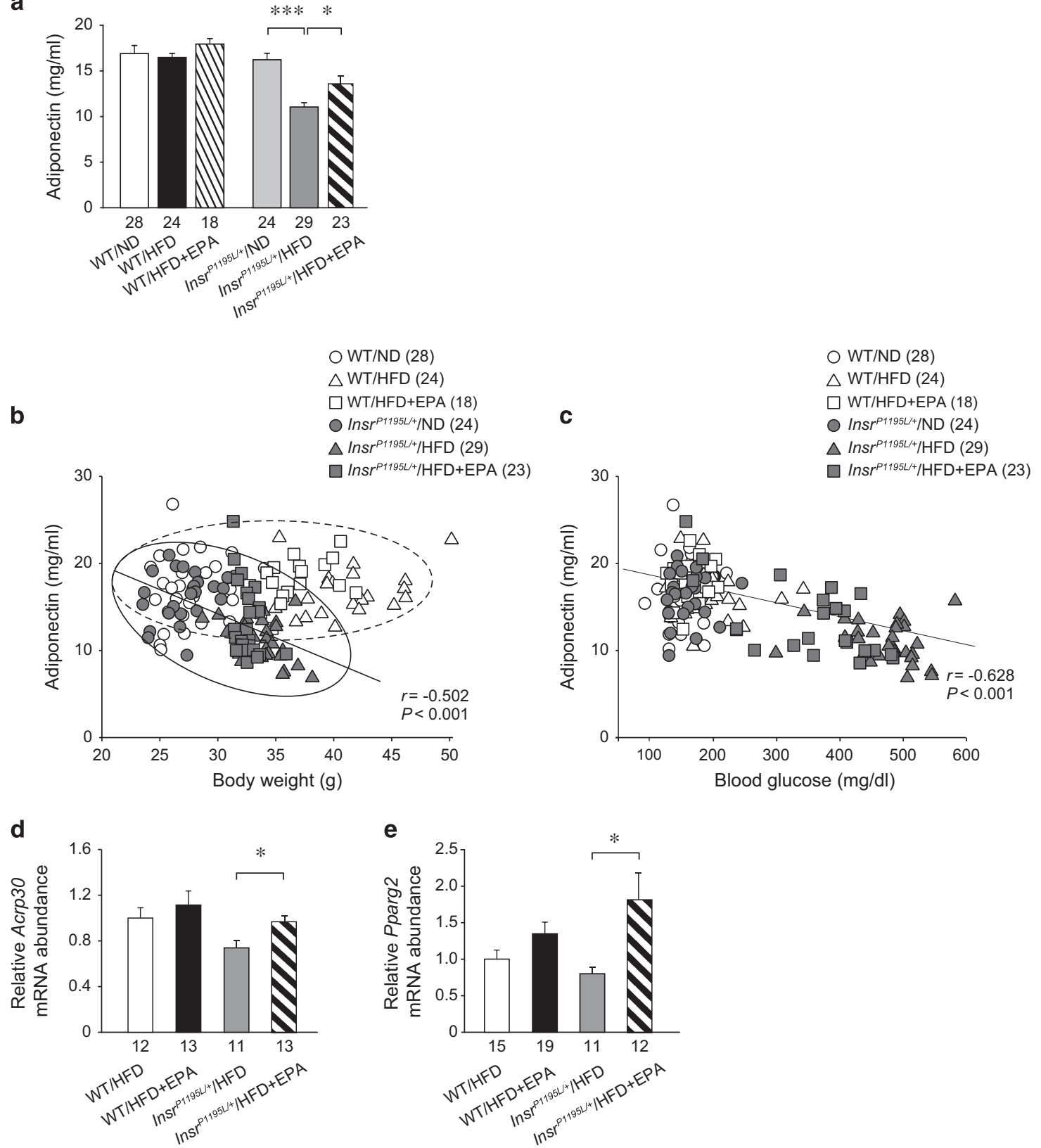

Figure 5. Serum adiponectin levels and their correlation with body weight or blood glucose levels. (a) Serum adiponectin levels fed ad libitum (16-18 weeks of age). (b) Relationship between body weight and adiponectin levels. Most of the data of Insr $P^{P 195 L /+}$ mice and WT mice are plotted inside the solid- and dotted-circle, respectively. (c) Relationship between blood glucose levels and adiponectin levels. (b, c) $r=$ Pearson's correlation coefficient. (d, e) mRNA expression levels of Acrp30 (d) and Pparg2 (e) in WAT under refed condition. Data are mean \pm s.e.m. ${ }^{*} P<0.05,{ }^{* * *} P<0.001$. The number in the bottom of the columns $(\mathbf{a}, \mathbf{d}, \mathbf{e})$ and in the parenthesis $(\mathbf{b}$, $\mathbf{c})$ denotes sample size.

EPA with a trend similar to that of adiponectin (Acrp30) mRNA (Figure 5e).

\section{DISCUSSION}

EPA is included in fish oil and there is a lot of epidemiological and experimental data showing that EPA has an inhibitory effect on cardiovascular diseases. ${ }^{15,16}$ In addition, EPA has been shown to decrease serum TG levels ${ }^{17}$ and inhibit platelet aggregation. ${ }^{18}$ However, the antidiabetic effect of EPA is still controversial. ${ }^{19}$

Our present results show that EPA treatment significantly improved blood glucose levels, glucose tolerance and insulin sensitivity in $I n s r^{P 1195 L /+} /$ HFD mice. We recently reported that HFD feeding induces hyperglycemia in $\operatorname{Insr}^{P 1195 L /+} / \mathrm{HFD}$ mice through increased lipolysis and increased gluconeogenesis from glycerol. ${ }^{3}$ Interestingly, abnormal upregulation of G6pc, the critical regulator of gluconeogenesis, was significantly ameliorated in the Insr ${ }^{P 1195 L /+} / \mathrm{HFD}$ liver by EPA supplementation. By contrast, EPA supplementation did not affect G6pc expression in the WT/HFD liver in accordance with the previous report in cafeteria diet-fed Wistar rats. ${ }^{20}$ Furthermore, EPA supplementation suppressed the increase in blood glucose levels after glycerol loading, which was shown to increase G6pc in the liver. ${ }^{3}$ These results suggest that EPA elicits its antidiabetic effect through the suppressed gluconeogenesis from glycerol. Considering that insulin suppresses G6pc expression in 
the liver, we examined whether EPA improves insulin resistance through its direct action on hepatocytes using primary hepatocytes from WT/HFD and InsP $P^{1195 L /+} /$ HFD mice. Our present studies in primary hepatocytes suggest that EPA might improve insulin sensitivity through a PI3K-independent pathway in InsP $^{P 1195 L /+} /$ HFD mice.

We then evaluated the effect of EPA on lipid metabolism in WAT. EPA has been reported to suppress chronic inflammation in WAT ${ }^{21}$ through various pathways, including activation of GPR120 and inhibition of Toll-like receptor $4 .^{22}$ Oh et al. ${ }^{7}$ reported that $n-3$ PUFA lessens insulin resistance through a signal involving GPR120 expressed in adipocytes and adipose tissue macrophages. Considering that EPA supplementation reduced Tnfa and Emr1 expression levels in WAT and serum FFA levels, anti-inflammatory action of EPA may contribute to the amelioration of hyperglycemia, at least in part, in $I n s r^{P 1195 L /+} /$ HFD mice.

Interestingly, EPA supplementation reduced adiposity in both

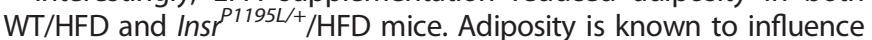
release of adipocytokines from WAT to mediate insulin sensitivity. ${ }^{23}$ Especially, adiponectin has been shown to improve insulin sensitivity in various insulin target tissues, such as liver and skeletal muscle, ${ }^{24}$ and to be decreased in obesity and T2DM. ${ }^{13}$ Nevertheless, Insr ${ }^{P 1195 L /+} /$ HFD mice exhibited lower adiponectin levels compared with those of WT/HFD mice despite the lower body weight. Although no correlation between body weight and adiponectin levels was detected in WT mice, there was a large decrease in adiponectin levels in response to body weight gain by HFD in Insr ${ }^{P 1195 L /+}$ mice. These results suggest that $\operatorname{Insr}^{P 1195 L /+}$ mice are susceptible to hypoadiponectinemia in response to body weight gain by HFD. By contrast, there was a clear negative correlation between blood glucose levels and adiponectin levels in Insr ${ }^{P 1195 L /+}$ mice. Notably, EPA supplementation improved hyperglycemia of $I n s r^{P 1195 L /+} /$ HFD mice along with the increase in adiponectin levels. In this study, a causal relationship between hypoadiponectinemia and hyperglycemia has not been shown. However, EPA supplementation increases mRNA expression of Pparg2, which has been reported to be transactivated through insulin. ${ }^{14}$ In addition, Oh et al. ${ }^{7}$ has reported that $n-3$ PUFA potentiates insulin signaling through Akt phosphorylation in adipocytes. Accordingly, EPA might improve insulin signaling in adipocytes of InsP ${ }^{P 195 L /+}$ /HFD mice, which could ameliorate their hypoadiponectinemia. Adiponectin has been reported to suppress gluconeogenesis through both AMPK signaling-dependent and -independent pathways. ${ }^{24,25}$ Accordingly, in the Insr ${ }^{P 1195 L /+} / \mathrm{HFD}$ liver, decreased adiponectin levels could elicit the increase in gluconeogenesis in the liver and lead to the development of hyperglycemia. Taken together, EPA supplementation is considered to act directly on hepatocytes to improve insulin signaling and indirectly to improve insulin signaling through enhancement of adiponectin secretion from WAT. In large clinical studies, EPA has been reported not to show an antidiabetic effect in human T2DM patients. ${ }^{26}$ However, the pathophysiology of T2DM is known to be heterogeneous. Our present study suggests that EPA supplementation may be effective in the treatment of T2DM patients with a pathophysiology similar to that of $I n s r^{P 1195 L /+} /$ HFD mice. Thus, considering personalized medicine to various T2DM patients, EPA supplementation might be one of the choices of therapeutics.

\section{CONFLICT OF INTEREST}

The authors declare no conflict of interest.

\section{ACKNOWLEDGEMENTS}

This work was supported by Grant-in-Aid from the Ministry of Education, Science, Sports, and Culture of Japan (23700900 and 16K00846 to E-YL and 25293051 and
16K08520 to TM). We thank Dr Takashi Yano (Mochida Pharmaceutical Co., Ltd.) for helpful advice during the course of the study.

\section{REFERENCES}

1 Nolan CJ, Damm P, Prentki M. Type 2 diabetes across generations: from pathophysiology to prevention and management. Lancet 2011; 378: 169-181.

2 Bhaswant M, Poudyal H, Brown L. Mechanisms of enhanced insulin secretion and sensitivity with n-3 unsaturated fatty acids. J Nutr Biochem 2015; 26: 571-584.

3 Lee EY, Sakurai K, Zhang X, Toda C, Tanaka T, Jiang M et al. Unsuppressed lipolysis in adipocytes is linked with enhanced gluconeogenesis and altered bile acid physiology in Insr ${ }^{\text {P1195L/+ }}$ mice fed high-fat-diet. Sci Rep 2015; 5: 17565.

4 Olefsky JM, Glass CK. Macrophages, inflammation, and insulin resistance. Annu Rev Physiol 2010; 72: 219-246.

5 Lee JY, Plakidas A, Lee WH, Heikkinen A, Chanmugam P, Bray G et al. Differential modulation of Toll-like receptors by fatty acids: preferential inhibition by $n-3$ polyunsaturated fatty acids. J Lipid Res 2003; 44: 479-486.

6 Arita M, Yoshida M, Hong S, Tjonahen E, Glickman JN, Petasis NA et al. Resolvin E1, an endogenous lipid mediator derived from omega-3 eicosapentaenoic acid, protects against 2,4,6-trinitrobenzene sulfonic acid-induced colitis. Proc Natl Acad Sci USA 2005; 102: 7671-7676.

7 Oh DY, Talukdar S, Bae EJ, Imamura T, Morinaga H, Fan W et al. GPR120 is an omega-3 fatty acid receptor mediating potent anti-inflammatory and insulinsensitizing effects. Cell 2010; 142: 687-698.

8 Miki T, Nagashima K, Tashiro F, Kotake K, Yoshitomi H, Tamamoto A et al. Defective insulin secretion and enhanced insulin action in KATP channeldeficient mice. Proc Natl Acad Sci USA 1998; 95: 10402-10406.

9 Kimura K, Yamada T, Matsumoto M, Kido Y, Hosooka T, Asahara S et al. Endoplasmic reticulum stress inhibits STAT3-dependent suppression of hepatic gluconeogenesis via dephosphorylation and deacetylation. Diabetes 2012; 61: 61-73.

10 Lee EY, Kaneko S, Jutabha P, Zhang X, Seino S, Jomori T et al. Distinct action of the alpha-glucosidase inhibitor miglitol on SGLT3, enteroendocrine cells, and GLP1 secretion. J Endocrinol 2015; 224: 205-214.

11 Perez-Matute P, Perez-Echarri N, Martinez JA, Marti A, Moreno-Aliaga MJ. Eicosapentaenoic acid actions on adiposity and insulin resistance in control and high-fat-fed rats: role of apoptosis, adiponectin and tumour necrosis factor-alpha. Br J Nutr 2007; 97: 389-398.

12 Murano I, Barbatelli G, Parisani V, Latini C, Muzzonigro G, Castellucci M et al. Dead adipocytes, detected as crown-like structures, are prevalent in visceral fat depots of genetically obese mice. J Lipid Res 2008; 49: 1562-1568.

13 Kadowaki T, Yamauchi T, Kubota N, Hara K, Ueki K, Tobe K. Adiponectin and adiponectin receptors in insulin resistance, diabetes, and the metabolic syndrome. J Clin Invest 2006; 116: 1784-1792.

14 Vidal-Puig A, Jimenez-Linan M, Lowell BB, Hamann A, Hu E, Spiegelman B et al. Regulation of PPAR gamma gene expression by nutrition and obesity in rodents. J Clin Invest 1996; 97: 2553-2561.

15 Yokoyama M, Origasa H, Matsuzaki M, Matsuzawa $Y$, Saito $Y$, Ishikawa $Y$ et al. Effects of eicosapentaenoic acid on major coronary events in hypercholesterolaemic patients (JELIS): a randomised open-label, blinded endpoint analysis. Lancet 2007; 369: 1090-1098.

16 Matsumoto M, Sata M, Fukuda D, Tanaka K, Soma M, Hirata Y et al. Orally administered eicosapentaenoic acid reduces and stabilizes atherosclerotic lesions in ApoE-deficient mice. Atherosclerosis 2008; 197: 524-533.

17 Musa-Veloso K, Binns MA, Kocenas AC, Poon T, Elliot JA, Rice $\mathrm{H}$ et al. Long-chain omega-3 fatty acids eicosapentaenoic acid and docosahexaenoic acid dose-dependently reduce fasting serum triglycerides. Nutr Rev 2010; 68: $155-167$.

18 Dyerberg J, Bang HO, Stoffersen E, Moncada S, Vane JR. Eicosapentaenoic acid and prevention of thrombosis and atherosclerosis? Lancet 1978; 2: 117-119.

19 Martinez-Fernandez L, Laiglesia LM, Huerta AE, Martinez JA, Moreno-Aliaga MJ. Omega-3 fatty acids and adipose tissue function in obesity and metabolic syndrome. Prostaglandins Other Lipid Mediat 2015; 121: 24-41.

20 Perez-Echarri N, Perez-Matute P, Marcos-Gomez B, Martinez JA, Moreno-Aliaga MJ. Effects of eicosapentaenoic acid ethyl ester on visfatin and apelin in lean and overweight (cafeteria diet-fed) rats. Br J Nutr 2009; 101: 1059-1067.

21 Chawla A, Nguyen KD, Goh YP. Macrophage-mediated inflammation in metabolic disease. Nat Rev Immunol 2011; 11: 738-749.

22 Talukdar S, Olefsky JM, Osborn O. Targeting GPR120 and other fatty acid-sensing GPCRs ameliorates insulin resistance and inflammatory diseases. Trends Pharmacol Sci 2011; 32: 543-550.

23 Tilg $\mathrm{H}$, Moschen AR. Adipocytokines: mediators linking adipose tissue, inflammation and immunity. Nat Rev Immunol 2006; 6: 772-783. 
24 Yamauchi T, Kamon J, Minokoshi Y, Ito Y, Waki H, Uchida S et al. Adiponectin stimulates glucose utilization and fatty-acid oxidation by activating AMPactivated protein kinase. Nat Med 2002; 8: 1288-1295.

25 Miller RA, Chu Q, Le Lay J, Scherer PE, Ahima RS, Kaestner $\mathrm{KH}$ et al. Adiponectin suppresses gluconeogenic gene expression in mouse hepatocytes independent of LKB1-AMPK signaling. J Clin Invest 2011; 121: 2518-2528.

26 Bosch J, Gerstein HC, Dagenais GR, Diaz R, Dyal L, Jung H et al. n-3 fatty acids and cardiovascular outcomes in patients with dysglycemia. N Engl J Med 2012; 367: 309-318.
This work is licensed under a Creative Commons Attribution 4.0 International License. The images or other third party material in this
article are included in the article's Creative Commons license, unless indicated otherwise in the credit line; if the material is not included under the Creative Commons license, users will need to obtain permission from the license holder to reproduce the material. To view a copy of this license, visit http://creativecommons.org/licenses/ by/4.0/

(c) The Author(s) 2016 